\title{
The influence of the typicality feature in the production of language associations in schizophrenia
}

\author{
Petra Dominik • Iva Kužina² • Vlasta Erdeljac ${ }^{2}$ Martina Sekulić Sović ${ }^{2}$ \\ - Ninoslav Mimica ${ }^{3,4}$ • Draženka Ostojićc ${ }^{3,5}$ • Aleksandar Savića 3,4 \\ 'University of Zadar, Postgraduate Doctoral Study, Programme in Humanities, ${ }^{2}$ University of \\ Zagreb, Faculty of Humanities and Social Sciences, Department of Linguistics, ${ }^{3}$ University \\ Psychiatric Hospital Vrapče, ${ }^{4}$ University of Zagreb, School of Medicine, Chair of Psychiatry and \\ Psychological Medicine, and ${ }^{5}$ University of Zagreb, Faculty of Law, Social Work Study Centre
}

https://doi.org/10.17234/9789531758314.08

\begin{abstract}
Language deficits in schizophrenia are hypothesized to be a result of increased spreading of activation in the semantic memory during language processing. The spreading of activation is determined by the features of concepts activated in search and retrieval processes. One of the features thought to have a great influence on processing is the typicality feature (Holmes \& Ellis, 2006). The aim of this study was to establish whether there are differences in language processing in schizophrenia subjects compared to a healthy control group in a verbal fluency task based upon the typicality feature. Specifically, the analysis was limited to the first cluster of the verbal fluency task because it requires the least effort in terms of task restrictions (Venneri et al., 2008). The study included 8 patients diagnosed with schizophrenia from the University Psychiatric Hospital Vrapče and 8 healthy control subjects. A category semantic fluency task was conducted on both groups, and the healthy control group was given a typicality rating task consisting of exemplars produced by both groups in the first cluster of the former task. Results analysis showed no differences in the typicality ratings of exemplars between the groups in any of the categories except the animals category.
\end{abstract}

Key words: schizophrenia language deficits, verbal fluency, associations, first cluster, typicality feature 


\section{Introduction}

Studies on the production and reception of language associations in schizophrenia are numerous as the use of idiosyncratic associations as well as a larger amount of direct and indirect associations is considered to be one of its symptoms. Among the first definitions of schizophrenia, Bleueler suggested loose associations as one of its main symptoms. In line with that, recent psycholinguistic literature has shown that subjects with schizophrenia use a larger amount of associations in their discourse (Maher et al., 2005; Elvevåg et al., 2007), idiosyncratic associations (Maher, 1983; McKenna \& Oh, 2005: 156-158; Johnson \& Shean, 1993), and in associative priming tests show a higher priming effect (Kreher et al., 2008). On account of the higher priming effect in associative priming in schizophrenia subjects, compared to healthy controls, Spitzer et al. (1993) concluded that subjects with schizophrenia show a faster spreading of activation and/or a lack of inhibition in the semantic memory during search and retrieval processes in language production. Other studies confirm the faster spreading activation with a lack of inhibition interpretation (Maher et al., 1987; Manschreck et al., 1988). Anderson (1983) proposed the theory of faster spreading activation in which the associations' connections and the speed of concept activation are important in the search processes. This theory presents concepts as hubs of associative elements and features and presupposes that associations are activated because of their connections, which can be semantic features, frequency, co-occurrences, etc. If search and retrieval processes arrive at an activated hub of features, which corresponds to an association, before the search reaches the actual target concept, the association can be produced if it is not correspondingly inhibited. In semantic memory models, spreading activation theories are prevalent in traditional cognitive models such as those presented by Quillian (1968) and elaborated by Collins and Loftus (1975). Quillian's model is hierarchical and supposes that concepts are hubs on different hierarchical aspects and that features are connected to a specific hierarchy level. Collins and Loftus elaborate on the model, claiming it is not hierarchical and that the activation spreads fluidly through concept nodes and hubs, depending upon features, connections, and concepts. The capacity and length of the activation are defined by different criteria, and the search is stopped when a sufficient level of activation is reached or by the passage of time. Collins and Loftus, who agree with this semantic memory model, assume that subjects with schizophrenia have faster spreading activation in which concepts are not appropriately inhibited after being activated before reaching the target word.

Studies on the structure and organization of the semantic memory offer information on associative structures that reflect concept representations. Because of this, they have been conducted since the beginning of interest in the semantic 
memory. Tulving (1972: 402) concluded that association tests offer a broad view of the semantic memory, and Cramer (1968) introduced them in psychology and linguistic studies Nelson et al. (2000) concluded that they offer a means of constructing the lexical-semantic knowledge of subjects who share the same cultural and language background. Along with free association tests, verbal fluency, lexical decision, priming, and scalar tests (among others) are conducted in association studies. The importance of verbal fluency tests is emphasized by the fact that they are decontextualized, while discourse production and statistical association analysis, for example, can also be contextualized.

Verbal fluency tasks require a subject's language production in assigned conditions and time restrictions. They differ on conditions; for example, there are semantic fluency tasks (listing examples of a specified semantic category), phonological fluency tests (listing examples starting with a same phoneme), and syntactic fluency tasks (listing examples with the same syntactic limitations). Semantic fluency tasks include activation of semantic memory concepts on account of semantic associative relations and include automatized search processes in a specific semantic subcategory (cluster) and controlled processes of transition from one subcategory to another (switching) (Troyer \& Moscovitch, 2006: 144).

At the start of the verbal fluency test, production depends on controlled and automatized processes and is restricted only by the restrictions of the task, but as time goes by, the subjects have to take into account further limitations: they need to remember all the produced exemplars and accessed subcategories to avoid repetition and returning to subcategories. Studies which analyzed production of 60 second verbal fluency tasks in 15 second intervals have concluded that the test follows a negative acceleration curve (Crowe, 1998).

It can be surmised that production in the first interval, and first cluster, is dependent mainly on the connectivity of the semantic store and lexical-semantic features of the concepts. Holmes and Ellis (2006) suggested that semantic typicality influences word-processing tasks in healthy adults, and Venneri et al. (2008) suggested that age of acquisition and semantic typicality for production in the first interval of the fluency tasks would be higher in schizophrenia patients when compared to healthy control subjects.

Semantic typicality refers to the degree to which a concept presents a certain semantic category (Rosch, 1975). Elvevåg et al. (2002) have concluded that semantic typicality has a great influence in categorization tests in subjects with schizophrenia. Juhasz et al. (2012) have concluded that schizophrenia subjects, in comparison with healthy control subjects, produce more typical category exemplars in earlier clusters of verbal fluency tasks, while Baskak et al. (2008) have concluded that schizophrenia subjects produce fewer typical category exemplars than healthy control subjects do. 
This study analyzes primary clusters, as they require the least effort in verbal fluency tasks (Crowe, 1998). Further cluster analysis was not taken into account because the goal of this study was to analyze the semantic typicality of category exemplars in subjects with schizophrenia in comparison with control subjects. Semantic typicality was established by a typicality rating task results analysis. An additional control group evaluated every produced concept of the primary cluster of both subject groups of the verbal fluency test on a 1-5 scale. Results of the typicality rating task of exemplars produced by subjects with schizophrenia were compared with the results of exemplars produced by the control group.

The goal of this study was to analyze semantic typicality of category exemplars in the first cluster of the verbal fluency task in subjects with schizophrenia in comparison with control subjects. Based on the typicality rating of the exemplars produced, patients produced less typical associations when compared to the healthy control group. The results for the patient group on typicality rating differed from the healthy controls depending on the category.

\section{Methods}

\subsection{Participants}

The study included eight patients from the University Psychiatric Hospital Vrapče, School of Medicine, University of Zagreb. Six patients had been diagnosed with acute schizophrenia-like psychotic disorder (F23.2), and two patients had diagnoses of acute polymorphic psychotic disorder with symptoms of schizophrenia (F23.1). The average time since the onset of the disorder was $1.5(\mathrm{SD}=2.73)$ months. All patients received antipsychotic therapy, and the average daily dose of antipsychotics expressed in chlorpromazine equivalents was 460.625 ( $S D=231.82$ ). Informed consent was obtained for all patients before the administration of the test, and the test was approved by the Ethics Committee of the University Psychiatric Hospital Vrapče (Registry number: 23-485/2-15). The average age of the patients was 25.375 ( $\mathrm{SD}=5.55) ; 6$ were female, 2 male, and all were right-handed. The healthy subjects for the first task were matched with the patients in age, handedness and sex. For the second test, the typicality rating task, another control group of healthy subjects, 42 Croatian-speaking volunteers with an average age of $26.6(\mathrm{SD}=2.51)$ years, were recruited.

\subsection{Materials and procedure}

The study consisted of two experiments, a semantic category verbal fluency test, and a typicality rating task. The semantic category fluency test was used in the first part of the study. The subjects were required to produce category exemplars in a restricted time frame of 60 seconds. The following categories were examined: 
animals, vehicles, beverages, clothes, trees, furniture, vegetables, fruits and musical instruments. All the categories were introduced by the examiner, through questions such as What animals can you think of? The replies were recorded by a voice recorder and later transcribed. The second experiment, the typicality rating task included exemplars produced by subjects in the first cluster of the verbal fluency task. The subjects made a subjective assessment of typicality of every word on a scale of 1 to 5 . This test was used to estimate words in relation to association prototype. The typicality rating task consisted of three associations forming the first cluster obtained from schizophrenia patients and healthy control subjects through the category fluency test. The participants were supposed to evaluate to what extent the task word is a prototype of a certain category. In addition to the listed associations, the experiment also contained brief instructions on how to evaluate the words from 1 to 5 in relation to the bolded word, written above the task word, representing the category: On a scale 1 to 5, where 1 represents not typical and 5 represents very typical, please judge how typical you consider this example to be as a prototype for its category.

\section{Results}

Differences between the healthy controls $(N=8)$ and the patients $(N=8)$ were tested for the first, the second, and the third association in the different categories of words (Table 1). The only difference was established for the second association of the category animals $(U(8,8)=8.00, z=2.52, p=.0117, r=.63)$, where the healthy controls had a higher typicality rating $(M d n=4.67, Q 1-Q 3=$ 4.51-4.75) than the patients ( $M d n=3.31, Q 1-Q 3=2.92-4.32)$.

Table 1. Results of Mann-Whitney $U$ test of the differences between the healthy controls and the patients

\begin{tabular}{|l|r|r|r|r|r|r|}
\hline & $\begin{array}{c}\text { Controls } \\
\text { Mdn }\end{array}$ & $\begin{array}{c}\text { Patients } \\
\text { Mdn }\end{array}$ & \multicolumn{1}{c|}{$U$} & \multicolumn{1}{c|}{$Z$} & \multicolumn{1}{c|}{-level } & \multicolumn{1}{c|}{$r$} \\
\hline Animals_first & 4.60 & 3.98 & 26.00 & 0.63 & .5286 & 0.16 \\
\hline Animals_second & 4.67 & 3.31 & 8.00 & 2.52 & $.0117^{*}$ & 0.63 \\
\hline Animals_third & 4.06 & 3.75 & 26.00 & 0.63 & .5286 & 0.16 \\
\hline Vehicles_first & 4.90 & 4.50 & 26.00 & 0.63 & .5286 & 0.16 \\
\hline Vehicles_second & 3.83 & 3.57 & 27.00 & 0.53 & .5995 & 0.13 \\
\hline Vehicles_third & 3.67 & 3.45 & 26.00 & 0.63 & .5286 & 0.16 \\
\hline Trees_first & 3.81 & 4.12 & 20.00 & -1.26 & .2076 & -0.32 \\
\hline Trees_second & 4.24 & 4.12 & 31.00 & -0.11 & .9164 & -0.03 \\
\hline Trees_third & 3.99 & 3.14 & 17.50 & 1.52 & .1278 & 0.38 \\
\hline Furniture_first & 4.43 & 4.69 & 18.00 & -1.47 & .1415 & -0.37 \\
\hline Furniture_second & 4.24 & 4.69 & 21.50 & -1.10 & .2701 & -0.28 \\
\hline
\end{tabular}




\begin{tabular}{|l|r|r|r|r|r|r|}
\hline & $\begin{array}{c}\text { Controls } \\
\text { Mdn }\end{array}$ & $\begin{array}{c}\text { Patients } \\
\text { Mdn }\end{array}$ & \multicolumn{1}{c|}{$U$} & \multicolumn{1}{c|}{$Z$} & \multicolumn{1}{c|}{$p$-level } & $r$ \\
\hline Furniture_third & 4.38 & 4.38 & 32.00 & 0.00 & 1.0000 & 0 \\
\hline Drinks_first & 4.81 & 4.36 & 16.50 & 1.63 & .1036 & 0.41 \\
\hline Drinks_second & 4.30 & 3.86 & 27.50 & 0.47 & .6365 & 0.12 \\
\hline Drinks_third & 3.99 & 3.85 & 29.00 & 0.32 & .7527 & 0.08 \\
\hline Vegetables_first & 4.43 & 4.05 & 13.50 & 1.94 & .0520 & 0.49 \\
\hline Vegetables_second & 4.21 & 4.39 & 25.00 & -0.74 & .4623 & -0.19 \\
\hline Vegetables_third & 4.43 & 4.43 & 27.00 & 0.53 & .5995 & 0.13 \\
\hline Clothes_first & 4.80 & 4.95 & 26.00 & -0.63 & .5286 & -0.16 \\
\hline Clothes_second & 4.67 & 4.95 & 15.50 & -1.73 & .0831 & -0.43 \\
\hline Clothes_third & 4.27 & 4.05 & 30.00 & 0.21 & .8336 & 0.05 \\
\hline Fruits_first & 4.86 & 4.48 & 19.00 & 1.37 & .1722 & 0.34 \\
\hline Fruits_second & 4.24 & 4.44 & 29.50 & -0.26 & .7929 & -0.07 \\
\hline Fruits_third & 3.55 & 4.24 & 28.50 & -0.37 & .7132 & -0.09 \\
\hline Instruments_first & 4.95 & 4.94 & 22.00 & 1.05 & .2936 & 0.26 \\
\hline Instruments_second & 3.74 & 3.99 & 23.50 & -0.89 & .3720 & -0.22 \\
\hline Instruments_third & 3.94 & 3.48 & 22.50 & 1.00 & .3184 & 0.25 \\
\hline
\end{tabular}

Legend: ${ }^{*} p<.05$

Table 2. Results of Mann-Whitney $U$ test measuring the differences between healthy controls and patients for different categories of words

\begin{tabular}{|l|r|r|r|r|r|r|}
\hline & $\begin{array}{c}\text { Controls } \\
M d n\end{array}$ & \multicolumn{1}{c|}{$\begin{array}{c}\text { Patients } \\
M d n\end{array}$} & \multicolumn{1}{c|}{$U$} & \multicolumn{1}{c|}{$Z$} & \multicolumn{1}{c|}{$p$-level } & \multicolumn{1}{c|}{$r$} \\
\hline Animals & 12.98 & 11.49 & 8.00 & 2.52 & $\mathbf{. 0 1 1 7 *}$ & 0.63 \\
\hline Vehicles & 12.54 & 11.58 & 25.00 & 0.74 & .4623 & 0.19 \\
\hline Trees & 12.30 & 11.84 & 20.00 & 1.26 & .2076 & 0.32 \\
\hline Furniture & 12.77 & 13.74 & 20.50 & -1.21 & .2271 & -0.30 \\
\hline Drinks & 12.73 & 11.96 & 27.00 & 0.53 & .5995 & 0.13 \\
\hline Vegetables & 12.89 & 12.18 & 16.00 & 1.68 & .0929 & 0.42 \\
\hline Clothes & 13.43 & 13.43 & 31.00 & 0.11 & .9164 & 0.03 \\
\hline Fruits & 12.64 & 12.85 & 30.50 & 0.16 & .8748 & 0.04 \\
\hline Instruments & 12.58 & 12.04 & 26.00 & 0.63 & .5286 & 0.16 \\
\hline
\end{tabular}

Legend: ${ }^{*} p<.05$

\section{Discussion}

The aim of this study was to analyze the production of language associations in subjects with schizophrenia, in relation to a healthy control group. Specifically, the aim was to analyze typicality of associations in the first cluster of the seman- 
tic fluency task, since typicality is considered to be one of the features that influence production in healthy and schizophrenic language processing the most, and the first cluster requires the least effort and is not dependent on further constraints of the task.

The leading hypothesis was not confirmed, as the schizophrenia subjects did not produce less typical associations when compared to the healthy control group. However, they did produce associations with a lower typicality rating in one of the analyzed semantic categories, the animals category. Animals are the only category containing the animacy feature, which, according to the CSA theory (Tyler \& Moss, 2001), is processed differently because of a higher amount of shared features in the semantic memory, unlike concepts without the animacy feature which are thought to be processes mostly on account of their distinctive features. Therefore, it is not unexpected to find the only relevant difference in the animals category.

Our results show us that, in all categories except one (furniture), the healthy control group achieved higher scores in typicality ratings of associations in the first cluster, although the difference was not statistically relevant $(p>0.5)$. The only statistically relevant difference was in the animals category, in which the patient group produced statistically less typical category exemplars. No other inter-group differences proved statistically relevant. This could be a result of the small subject sample. Nevertheless, although the rest of the tested categories show no statistically relevant inter-group differences, there is a tendency toward the production of less typical exemplars in the patient group, which could be statistically relevantly reproduced on a larger subject sample.

The results obtained from the two tests (the semantic category fluency task and the typicality scale task) have shown differences between the two subject groups only in the animals category, while there were no differences in the rest of the semantic categories. These results are not in line with either Juhasz et al's (2012) or Baskak et al.s (2008) results, who found differing typicality rating results in the first cluster for their schizophrenia subject groups when compared to healthy control groups. The reason for this could be the small sample of the present study, but also the fact that the patients examined were first-episode psychosis patients early in the course of their illness, and the typicality rating could be a "state marker," changing across the phases of the illness.

These results also indicate differences in the language processing of the animals category and could suggest differences in the processing of animacy features. Additionally, the results show that there are no differences in language processing of the typicality feature in other semantic categories in first-episode psychosis subjects in relation to the healthy control group. Future studies should additionally analyze the processing of animacy features and typicality in schizophrenia-spectrum disorders across different illness phases. 


\section{Conclusion}

The results of this study were analyzed in accordance with current psycholinguistic theories, but further studies are needed on a larger subject sample, although the statistical analysis provided clear results.

This study chose to analyze only the first cluster of verbal fluency task as it is thought to be the cluster most dependent on the automatic processes of language production, since each subsequent cluster demands further task restrictions which are considered to be under the controlled processes domain. Hence, the first cluster relies the most on automatic processes, i.e. on the connectivity of the semantic memory and the lexical-semantic features of the concepts which form it. As the goal of this study was to analyze the influence of the typicality feature, the analysis of the first cluster of production proved to be the most relevant. Analysis of the whole production on the fluency task by intervals could prove valuable and remains an interesting suggestion for further studies.

In this study, the typicality rates were provided by the healthy control group; one possibility for a follow-up study would be to compare the typicality ratings of both subject groups, so as to further establish differences between the groups' lexical-semantic processing of both associations and the typicality feature.

\section{References}

Anderson, J. R. (1983). A spreading activation theory of memory. Journal of Verbal Learning \& Verbal Behavior, 22(3), 261-295.

Baskak, B., Ozel, E. T., Atbasoglu, E. C., \& Baskak, S. C. (2008). Peculiar word use as a possible trait marker in schizophrenia. Schizophrenia Research, 103, 311-317.

Collins, A. M., \& Loftus, E. F. (1975). A spreading-activation theory of semantic processing. Psychological Review, 82(6), 407-428.

Crowe, S. F. (1998). Decrease in performance on the Verbal Fluency Test as a function of time: Evaluation in a young healthy sample. Journal of Clinical and Experimental Neuropsychology, 20(3), 391-401.

Elvevåg, B., Foltz, P. W., Weinberger, D. R., \& Goldberg, T. E. (2007). Quantifying incoherence in speech: An automated methodology and novel application to schizophrenia. Schizophrenia Research, 93(1-3), 304-316.

Elvevåg, B., Weickert, T., Wechsler, M., Coppola, R., Weinberger, D.R., \& Goldberg, T.E. (2002). An investigation of the integrity of semantic boundaries in schizophrenia. Schizophrenia Research, 53, 187-198.

Fernaeus, S. E., \& Almkvist, O. (1998). Word production: Dissociation of two retrieval modes of semantic memory across time. Journal of Clinical and Experimental Neuropsychology, 20(2), 137-143. 
Holmes, S. J., \& Ellis, A. W. (2006). Age of acquisition and typicality effects in three object processing tasks. Visual Cognition, 13, 884-910.

Johnson, D. E., \& Shean, G.D. (1993). Word associations and schizophrenic symptoms. Journal of Psychiatric Research, 27(1), 69-77.

Juhasz, B. J., Chambers, D., Shesler, L. W., Haber, A., \& Kurtz, M. M. (2012). Evaluating lexical characteristics of verbal fluency output in schizophrenia. Psychiatry Research, 200(2-3), 177-183.

Kreher, D. A., Holcomb, P. J., Goff, D., \& Kuperberg, G. R. (2008). Neural evidence for faster and further automatic spreading activation in schizophrenic thought disorder. Schizophrenia Bulletin, 34(3), 473-482.

Maher, B. A., Manschreck, T. C., Linnet, J., \& Candela, S. (2005). Quantitative assessment of the frequency of normal associations in the utterances of schizophrenia patients and healthy controls. Schizophrenia Research, 78(2-3), 219-224.

Maher, B., Manschreck, T. C., Hoover, T. M., \& Weisstein, C. C. (1987). Thought disorder and measured features of language production. In P. D. Harvey \& E. E. Walker (Eds.), Positive and negative symptoms in psychosis (pp. 195-215). Hillsdale, NJ: Erlbaum Associates.

Manschreck, T. C., Maher, B. A., Milavetz, J. J., Ames, D., Weisstein, C. C., \& Schneyer, M. L. (1988). Semantic priming in thought disordered schizophrenic patients. Schizophrenia Research, 1(2), 61-66.

McKenna, P., \& Oh, T. (2005). Schizophrenic speech: Making sense of bathroots and ponds that fall in doorways. Cambridge: Cambridge University Press.

Nelson, D. L., McEvoy, C. L., \& Dennis, S. (2000). What is free association and what does it measure? Memory and Cognition, 28(6), 887-899.

Quillian, M. (1968). Semantic memory. In M. Minsky (Ed.), Semantic information processing (pp. 227-270). Cambridge, MA: MIT Press.

Rosch, E. (1975). Cognitive representations of semantic categories. Journal of Experimental Psychology: General, 104, 192-233.

Spitzer, M., Braun, U., Hermle, L., \& Maier, S. (1993). Associative semantic network dysfunction in thought-disordered schizophrenic patients: Direct evidence from indirect semantic priming. Biological Psychiatry 34(12), 864-877.

Troyer, A. K., \& Moscovitch, M., (2006). Cognitive processes of verbal fluency tasks. In: E. M. Poreh (Ed.), Studies on neuropsychology, neurology and cognition: The quantified process approach to neuropsychological assessment (pp. 143-160). Philadelphia, PA: Taylor \& Francis.

Tulving, E., \& Donaldson, W. (1972). Organization of memory. New York, NY, London, UK: Academic Press.

Tyler, L. K., \& Moss, H. E. (2001). Towards a distributed account of conceptual knowledge. Trends in Cognitive Sciences, 5, 244-252.

Venneri, A., McGeown, W. J., Hietanen, H. M., Guerrini, C., Ellis, A. W., \& Shanks, M. F. (2008). The anatomical bases of semantic retrieval deficits in early Alzheimer's disease. Neuropsychologia, 46, 497-510. 